\title{
The Feasibility and Impact of a Yoga Intervention on Cognition, Physical Function, Physical Activity, and Affective Outcomes Among People Living with HIV: A Randomized Controlled Pilot Trial
}

Adria Quigley ( $\square$ adriaquigley@gmail.com )

Dalhousie University https://orcid.org/0000-0003-2404-7949

\section{Marie-Josée Brouillette \\ McGill University \\ Jacqueline Gahagan \\ Dalhousie University}

Kelly K. O'Brien

University of Toronto

Marilyn MacKay-Lyons

Dalhousie University

\section{Research Article}

Keywords: HIV, AIDS, Yoga, Cognition, Balance

Posted Date: April 15th, 2020

DOI: https://doi.org/10.21203/rs.3.rs-23012/v1

License: (c) (1) This work is licensed under a Creative Commons Attribution 4.0 International License. Read Full License

Version of Record: A version of this preprint was published at Journal of the International Association of Providers of AIDS Care (JIAPAC) on January 1st, 2020. See the published version at https://doi.org/10.1177/2325958220935698. 


\section{Abstract}

The purpose of this pilot randomized controlled trial was to assess the feasibility and impact of a tri-weekly 12-week yoga intervention among people living with HIV (PLWH). Additional objectives included evaluating cognition, physical function, medication adherence, health-related quality of life (HRQoL), and mental health among yoga participants versus controls using blinded assessors. We recruited 22 medically-stable PLWH aged $\geq 35$ years. $A$ priori feasibility criteria were $\geq 70 \%$ yoga session attendance and $\geq 70 \%$ of participants satisfied with the intervention using a post-participation questionnaire. Two participants withdrew from the yoga group. Mean yoga class attendance was $82 \%$ with $100 \%$ satisfaction. Intention-to-treat analyses (yoga $n=11$, control $n=11$ ) showed no withinor between-group differences in cognitive and physical function. The yoga group improved over time in HRQoL cognition $(p=.047)$ with trends toward improvements in HRQoL health transition $(p=.063)$ and depression $(p=.055)$. This pilot study provides preliminary evidence of feasibility and benefits of yoga for PLWH.

\section{Background}

The most recent estimates indicate that 36.9 million people worldwide are living with HIV (Human Immunodeficiency Virus). 1 The highest percentage of people living with HIV (PLWH) is in Sub-Saharan Africa, having 53\% of the global prevalence. 2 Those at risk of HIV transmission include women, men who have sex with men, transgender individuals, people who use drugs, and sex workers.3

Approximately 15-69\% of people living with HIV (PLWH) world wide experience some form of cognitive impairment,4-7 presenting as deficits in attention, memory, and executive function.8,9 There are sex differences in cognitive performance among PLWH, with women scoring lower on tests of attention, processing speed, executive function, and fine motor performance than men.10 In addition to cognitive impairment, balance and gait deficits are common among PLWH11 and are associated with elevated falls risk and mortality.12 Some evidence has emerged to indicate that a relationship exists between physical and cognitive performance in this population.13

Mind-body exercise is a type of therapy, such as Tai Chi and yoga that includes concentration, breathing, and body movement.14 These interventions can have a positive effect on cognitive performance among older adults without HIV. A recent meta-analysis of 32 randomized controlled trials (RCTs) evaluated mind-body interventions (of which, 8 RCTs included a yoga intervention), showing significant improvements in overall cognitive performance, working memory, verbal fluency, cognitive flexibility, and learning among older adults.14 Another meta-analysis of 11 RCTs (4 RCTs with yoga interventions) conducted with older adults determined that mind-body exercise had a positive effect on overall cognition, memory, executive function, learning, and language.15 A prominent theory explaining these cognitive benefits with yoga includes the down-regulation of the stress response and up-regulation of the parasympathetic nervous system.16,17 No RCTs to date have evaluated the impact of a yoga intervention on cognitive performance outcomes among PLWH.

Over half of PLWH in Western countries have sought complementary or alternative therapies, which include mindbody interventions such as yoga and Tai Chi.18-20 Some authors have evaluated the impact of Tai Chi on healthrelated quality of life and mental health in this population. A single group observational study conducted with male $(n=35)$ and female $(n=24)$ PLWH reported a positive effect of Tai Chi on HIV-related psychological distress in addition to emotional, social, and overall health-related quality of life.21 An RCT of 38 male PLWH living with AIDS (Acquired ImmunoDeficiency Syndrome) conducted by Galantino and colleagues (2005) in the United States found significant improvements in overall health-related quality of life in both aerobic exercise and Tai Chi groups versus controls. 22 
RCTs and meta-analyses have also demonstrated positive effects of yoga on affective outcomes such as healthrelated quality of life and mental health among PLWH. An RCT demonstrated significant improvements in anxiety, depression, fatigue, well- being, and quality of life in a sample of mostly female PLWH who participated in an integrated yoga intervention compared to controls following an 8-week intervention.23 Another RCT demonstrated positive effects on health-related quality of life and mental health among mostly female PLWH following a 12-week Sudarshan Kriya yoga intervention compared to controls.24 However, the intervention did not include a physical activity component, and PLWH taking antiretrovirals were excluded from the study.24 An RCT with a 1-month integrated yoga intervention conducted with male $(n=24)$ and female $(n=20)$ PLWH showed a significant reduction in depression scores among yoga participants compared to controls.25 A meta-analysis of 7 RCTs conducted with PLWH determined that yoga interventions resulted in large improvements in perceived stress, positive affect, and anxiety compared to controls.26 Finally, a systematic scoping review of 84 studies showed that mindfulness, relaxation techniques, cognitive-behavioral strategies, and yoga had beneficial effects on health-related quality of life and physical and psychological symptoms among PLWH.18

Emerging evidence also suggests that mind-body exercise can improve physical performance among PLWH. Participants in the abovementioned RCT by Galantino and colleagues (2005) who performed aerobic exercise and Tai Chi had significant improvements in balance using the functional reach test.22 Kietrys and colleagues (2018) also observed improvements in gait and balance with their integrative yoga intervention among two of three caseseries participants. 27 Yoga has the potential to address both physical and cognitive impairments in this population. However, no RCTs to date have evaluated the feasibility and impact of yoga on cognitive and physical outcomes among PLWH.

Women are frequently under-represented in health research, particularly in HIV trials.28 To address this gap, the Sex and Gender Equity in Research (SAGER) guidelines recommend authors report how sex (biological attributes) and gender (socially-constructed roles, behaviours, and identity) factor into the study design and present study data disaggregated by sex and gender if possible.29 As such, we will discuss and evaluate the role of sex and gender in this study where applicable.

\section{Purpose, Objectives, \& Hypothesis}

Our overall goal was to inform the design of a future, full-scale, multisite, community- based RCT to evaluate the effects of yoga on cognitive and physical function among PLWH from Halifax, Nova Scotia, Canada. Our primary objective was to assess the feasibility of a 12- week yoga intervention for PLWH in terms of participant recruitment, assessments, safety, adherence, and satisfaction with the intervention. Our second objective was to evaluate the effect of a 12-week yoga intervention (yoga group) versus usual care (control group) on cognitive performance and self-reported cognition among PLWH. Our third objective was to compare the effects of the yoga intervention versus control on balance, walking speed, mental health, medication adherence, physical activity, and health-related quality of life outcomes among PLWH. The fourth objective was to explore the relationships between cognitive performance and self-reported cognition, physical activity, and balance performance. Our alternative hypotheses were: 1 . The yoga group would experience larger improvements in cognitive performance and self-reported cognition than the control group and 2. The yoga group would experience larger improvements in physical function, health-related quality of life, and mental health outcomes than the control group.

\section{Methods}


We designed a pilot RCT. The Consolidated Standards of Reporting Trials (CONSORT) checklist for pilot and feasibility studies30 is shown in appendix 1.

\section{Participants}

The CONSORT31 diagram in Figure 1 shows the flow of participants through this randomized, two-parallel group, pilot trial. Participants were included in the study if they were aged 35 years or older, identified cognitive concerns on the Communicating Cognitive Concerns Questionnaire (C3Q) with a cut-off of 35 points or less at baseline,32 lived in the Halifax Regional Municipality of Nova Scotia, Canada, were able to provide informed consent, and were medically stable as determined by the Physical Activity Readiness Questionnaire Plus.33 PLWH already participating in regular yoga classes were excluded. We recruited participants

from community and health organizations in Halifax, Canada using posters, newsletters, and clinic staff assistance. We attempted to obtain a diverse sample in terms of ethnicity and gender by using snowball sampling techniques. [Insert Figure 1]

\section{Study procedures}

Study procedures were in accordance with institutional ethical standards and the Helsinki Declaration (2013). The study methods are described in detail elsewhere.34 In summary, individuals wanting to participate contacted the study coordinator who conducted a screening assessment to confirm eligibility, explained the study's purpose, procedures, risks and benefits, and time responsibilities of the study, reviewed the consent form, and answered questions. Those who agreed to participate in the study signed the consent form and underwent the baseline assessment. Thereafter, participants were randomly assigned in a 1:1 ratio to the yoga or control group. Group assignment was determined by a random computer generator and was concealed in individual, opaque envelopes by a person not affiliated with the study. The study coordinator opened the envelopes after completion of the baseline assessment. Assessors blinded to group allocation conducted the post-intervention assessment 12 weeks later. We instructed participants to avoid revealing their group allocation to assessors. To determine the effectiveness of the blinding process, we asked the assessors at study completion if they knew the group assignment of participants, and if they did not know, to guess the group to which the participant had been assigned.

\section{Intervention protocols}

Participants in the yoga group engaged in group-based Hatha yoga classes for 60 minutes, 3 times weekly for 12 weeks at a yoga studio. Hatha yoga has many variants forms of yoga including lyengar, Vinyasa, and Ashtanga35 with considerable variation between and within types.36 Our yoga program incorporated common components of Hatha yoga: asanas (physical poses or postures), meditation (dhyana), breathing exercises (pranayama), the study of introspection or self-study (swadhyaya), and spiritual discourse (satsang).35,37 Classes began with 15 minutes of seated meditation, breathing exercises, shoulder, neck and back stretches, and sun salutations (either in seated or standing). This was followed by 10 minutes of standing poses, 15 minutes of balance poses, and 10 minutes of abdominal and back-bend poses. All classes finished with 10 minutes of cool-down stretches and final rest (savasana). Classes were adapted to all ability levels by providing modifications to physical postures and assistive props. At the yoga classes, participants were provided with yoga mats, chairs, bolsters, blocks, straps, and blankets. To honour the Mi'kmaq peoples, on whose ancestral land the classes were conducted, an Indigenous Elder 
performed brief monthly smudging ceremonies [the burning of plants such as sage or sweetgrass for purification38] at the yoga studio. Participants were not explicitly encouraged to practice at home. We directed control participants to maintain their usual physical activity levels during the study.

Participants were given $\$ 50$ honoraria at baseline and final assessments. We offered bus tickets and parking reimbursements for assessments and bus tickets for those attending the yoga classes. We provided child-care reimbursement to those in the yoga group to address participation barriers, which are prevalent among female participants.39

\section{Outcome measures}

We assessed the feasibility of the trial by measuring participant recruitment, assessments, safety, adherence, and satisfaction with the intervention. Participant recruitment measures (including reasons for not participating and recruitment time), assessment duration, adverse events, and adherence (number of yoga classes attended) were recorded. An a priori target for adherence to determine whether to proceed with a large-scale RCT was that participants attend at least $70 \%$ of the yoga classes. The post-participation satisfaction questionnaire consisted of 7 Likert scale questions ranging from strongly disagree (1) to strongly agree (5) and 6 open-ended questions. The $a$ priori target for participant satisfaction was that a minimum of $70 \%$ of participants would indicate they were satisfied with the intervention, as determined by achieving scores of $\geq 4$ on the Likert scale for each item on the postparticipation questionnaire. The questionnaire was administered to yoga participants at study completion.

Study assessments included a demographic questionnaire administered at baseline (age, gender, ethnicity, education, employment, CD4+ cell count, viral load, time since HIV diagnosis, number of comorbidities, and antiretroviral use). We administered the following performance- based measures and paper-based self-reported questionnaires at baseline and 12 weeks: the Brief Cognitive Ability Measure (B-CAM),40,41 a computerized measure of cognitive performance with higher scores reflecting better cognitive ability; the Communicating Cognitive Concerns Questionnaire (C3Q),32 an HIV-specific self-reported cognitive questionnaire with higher scores indicating fewer cognitive difficulties; Community Balance and Mobility Scale (CB\&M),42 a measure of dynamic balance performance where higher scores indicate better balance performance; the 10-metre walk test, an objective test of comfortable and fast gait speed;43 the Rapid Assessment of Physical Activity (RAPA) questionnaire, a two-part, selfreported questionnaire where RAPA 1 indicates aerobic physical activity levels and the RAPA 2 indicates resistance and flexibility activities;44 accelerometer data $\left(\right.$ Fitbit $\left.^{\mathrm{TM}}\right)$ including steps per day and number of kilometers walked;45 the Simplified Medication Adherence Questionnaire (SMAQ), a self-reported antiretroviral medication adherence measure;46 the Medical Outcomes Survey-HIV (MOS-HIV) a self-reported health-related quality of life questionnaire developed for PLWH with 11 subscales (physical function, social function, role function, cognitive function, pain, mental health, energy, health distress, quality of life, general health, and health transition);47 and the Hospital Anxiety and Depression Scale (HADS-A and -D), a self-reported mental health questionnaire.48

\section{Data collection and analysis}

Study data were collected at Dalhousie University in Halifax, Nova Scotia, and were recorded using Research Electronic Data Capture (REDCap) software.49 The authors will provide access to the data if requested. All outcomes were assessed for missing data. Results were analysed and reported by gender, as recommended by the SAGER 
guidelines.29 Although sample size calculations are not recommended for pilot studies,30 we targeted a sample size of 25 participants in total in order to inform future sample size calculations.

\section{Primary outcomes}

We analyzed Likert post-intervention questionnaire responses descriptively, and open- ended responses using inductive thematic analysis. 50

\section{Descriptive statistics}

Means, mean change, and 95\% confidence intervals were calculated separately for the intervention and control groups as per the CONSORT guidelines.30 Floor and ceiling effects were assessed and calculated for the CB\&M by taking the proportion of the sample that scored the minimum or maximum scores.

\section{Second and third outcomes}

We used intention to treat and per-protocol approaches for our second and third outcome analyses. Spearman correlation coefficients were used to evaluate the relationship between outcomes with 0.1-0.3 representing a weak associations, $0.4-0.6$ representing moderate correlations, and $0.7-0.9$ representing strong associations. 51 We performed independent t-tests, Chi square, or Fisher's exact tests on demographic variables and baseline dependent measures, as appropriate.

We evaluated the data to determine whether they met the assumptions for parametric tests. If the assumptions for parametric tests were not met, we used non-parametric tests or bootstrapping. Bootstrapping is a non-parametric approach to hypothesis testing that is recommended for small samples that do not meet the assumption of normality.52,53 This process generates an estimate of the sampling distribution of a statistic (such as the standard error or confidence interval) by performing repeated random re-sampling from the available data and estimating bias-corrected and accelerated coefficients and p-values.52,53 We then analyzed secondary and tertiary outcomes using univariate analysis of covariance (ANCOVA) with bootstrapped confidence intervals, mixed ANCOVA, or Wilcoxon Signed Rank tests. Two-sided tests with a minimum alpha level of.05 were used for all analyses using SPSS Version 25 (IBM, 2017).54 We calculated effect sizes using partial eta squared for parametric and $r$ for nonparametric tests with 0.1 representing a small effect, 0.3 considered a moderate effect, and 0.5 representing a large effect.55-57 We also conducted a separate analysis with gender as a covariate to determine its effect on all secondary and tertiary outcomes.

\section{Supplementary analyses (fourth objective)}

We performed a moderator analysis to determine the effect of the interaction between baseline cognitive and balance performance on cognitive performance at study completion.

\section{Ethical approval and informed consent}


We obtained ethics approval through the Nova Scotia Health Authority (protocol reference \#1022158) and registered on clinicaltrials.gov (NCT03071562). Written informed consent was obtained prior to enrollment in the study.

\section{Results}

\section{Sample characteristics}

The characteristics of the sample are summarized in Table 1.

Table 1: Characteristics of the sample at baseline ( $\mathrm{n}=22$ participants)

\begin{tabular}{|c|c|c|c|c|}
\hline Characteristic & $\begin{array}{l}\text { Total sample } \\
(\mathrm{n}=22)\end{array}$ & $\begin{array}{l}\text { Yoga condition } \\
(\mathrm{n}=11)\end{array}$ & $\begin{array}{l}\text { Control condition } \\
(\mathrm{n}=11)\end{array}$ & $\begin{array}{l}\text { P-value (baseline } \\
\text { differences) }\end{array}$ \\
\hline \multicolumn{5}{|l|}{ Demographic characteristics } \\
\hline Age (years), (mean, SD) & $55.5(10.7)$ & $50.7(10.2)$ & $60.2(9.2)$ & $.034^{*}$ \\
\hline $\begin{array}{l}\text { Gender, n (\%) } \\
\text { Male } \\
\text { Female } \\
\text { Two-spirited } † \\
\text { Transgender }\end{array}$ & $\begin{array}{l}15(68.2 \%) \\
5(22.7 \%) \\
2(9.1 \%) \\
0(0 \%)\end{array}$ & $\begin{array}{l}9(81.8 \%) \\
1(9.1 \%) \\
1(9.1 \%) \\
0(0 \%)\end{array}$ & $\begin{array}{l}6(54.5 \%) \\
4(36.4 \%) \\
1(9.1 \%) \\
0(0 \%)\end{array}$ & .301 \\
\hline $\begin{array}{l}\text { Ethnicity, n (\%) } \\
\text { Caucasian } \\
\text { Indigenous } \\
\text { Mixed ethnicity } \\
\text { Black }\end{array}$ & $\begin{array}{l}16(72.7 \%) \\
3(13.6 \%) \\
2(9.1 \%) \\
1(4.5 \%)\end{array}$ & $\begin{array}{l}9(81.8 \%) \\
2(18.2 \%) \\
0 \\
0\end{array}$ & $\begin{array}{ll}7 & (63.6 \%) \\
1 & (9.1 \%) \\
2 & (18.2 \%) \\
1 & (9.1 \%)\end{array}$ & .310 \\
\hline $\begin{array}{l}\text { Education, n (\%) } \\
\text { Some high school } \\
\text { High school diploma } \\
\text { Some university } \\
\text { University degree }\end{array}$ & $\begin{array}{l}3(13.6 \%) \\
10(45.5 \%) \\
2(9.1 \%) \\
7(31.8 \%)\end{array}$ & $\begin{array}{l}1(9.1 \%) \\
4(36.4 \%) \\
2(18.2 \%) \\
4(36.4 \%)\end{array}$ & $\begin{array}{l}2(18.2 \%) \\
6(54.5 \%) \\
0 \\
3(27.3 \%)\end{array}$ & .411 \\
\hline $\begin{array}{l}\text { Employment, n (\%) } \\
\text { Unable to work or looking for work } \\
\text { Retired or student } \\
\text { Employed for wages full-time } \\
\text { Employed for wages part-time }\end{array}$ & $\begin{array}{l}10(45.5 \%) \\
5(22.7 \%) \\
3(13.6 \%) \\
4(18.2 \%)\end{array}$ & $\begin{array}{l}5(45.5 \%) \\
1(9.1 \%) \\
2(18.2 \%) \\
3(27.3 \%)\end{array}$ & $\begin{array}{l}5(45.5 \%) \\
4(36.4 \%) \\
1(9.1 \%) \\
1(9.1 \%)\end{array}$ & .372 \\
\hline \multicolumn{5}{|l|}{ Disease-related characteristics } \\
\hline CD4+ (cells/mm3) (mean, SD) & $515.5(231.4)$ & $552.9(184.5)$ & $482.2(273.2)$ & .547 \\
\hline $\begin{array}{l}\text { Number of other health } \\
\text { conditions (mean, SD) }\end{array}$ & $2.09(2.49)$ & $2.09(3.02)$ & $2.09(1.97)$ & 1.000 \\
\hline Years living with HIV (mean, SD) & $20.2(13.0)$ & $15.1(12.1)$ & $25.3(12.3)$ & .064 \\
\hline $\begin{array}{l}\text { Viral load, } \mathrm{n}(\%) \\
\text { Undetectable } \\
\text { Detectable }\end{array}$ & $\begin{array}{l}16(84.2 \%) \\
3(15.8 \%)\end{array}$ & $\begin{array}{l}8(88.9 \%) \\
1(11.1 \%)\end{array}$ & $\begin{array}{l}8(80 \%) \\
2(20 \%)\end{array}$ & 1.000 \\
\hline $\begin{array}{l}\text { Taking antiretrovirals, n (\%) } \\
\text { Yes } \\
\text { No }\end{array}$ & $\begin{array}{l}19(86.4 \%) \\
3(13.6 \%)\end{array}$ & $\begin{array}{l}10(90.9 \%) \\
1(9.1 \%)\end{array}$ & $\begin{array}{l}9(81.8 \%) \\
2(18.2 \%)\end{array}$ & 1.000 \\
\hline
\end{tabular}

*Significant difference $(\mathrm{p}<.05)$. Undetectable viral load is defined as $<50$ copies of HIV per milliliter of blood. SD, standard deviation

†Refers to Indigenous people who identify fluid gender roles and sexuality beyond the Western binaries of male/female and homosexual/heterosexual58

Participants were on average 55.5 years old $(S D=10.7)$ and had been living with HIV for a mean of 20.1 years $(S D=$ 13.0). Most participants (86\%) were taking antiretrovirals, were virologically suppressed (84\%), and were not immunocompromised, with a mean CD4+ count of 515.5 (SD = 231.4). Participants mostly identified as male $(68 \%)$ and Caucasian (73\%), with some education (59\% had some high school education or a high school diploma), and were not currently working (68\% were not working, retired, or studying). The two groups differed at baseline in cognitive performance (B-CAM) and age, therefore these variables were included as covariates to all parametric analyses to control for group imbalances.

\section{Feasibility outcomes (first objective)}


We approached 29 individuals to participate in the study. All were eligible and $22(76 \%)$ agreed to participate over a 23-month recruitment period. Five individuals gave no reason for declining to participate, one person did not wish to practice yoga, and one individual reported a lack of time. We did not achieve our initial goal to reach 25 participants, as recruitment slowed during the summer months and Halifax is a small community. The blinded assessors reported that they were unaware of group allocation of any participant at follow up.

Assessments took less than 2 hours to complete and study assessors deemed them to be feasible. One minor musculoskeletal injury occurred as a result of the balance assessment. There were no known injuries associated with the yoga intervention. Two participants (9\%) withdrew from the yoga group. Reasons for drop out included illness and relocating out of province. None of the participants withdrew from the control group. Mean attendance to total yoga classes was 82\% (mean attendance $=29.4 / 36$ classes), and 89\% (8/9) of participants in the yoga group met the a priori adherence criterion. One participant was unable to attend some classes at the yoga studio; therefore, the participant was offered taped or live virtual classes, which the participant accepted.

Nine participants had difficulties syncing their accelerometers as many did not have access to smartphones or computers. As a result, the study coordinator met with participants to sync their devices with a study computer.

\section{Quantitative Satisfaction Questionnaire Responses}

From the 9 participants in the yoga group, 100\% enjoyed the sessions, 100\% felt there was some benefit from participating in yoga, $100 \%$ felt safe during the sessions, and $100 \%$ agreed or strongly agreed that they felt comfortable during the yoga sessions. One minor musculoskeletal injury occurred as a result of the balance assessment. There were no known injuries associated with the yoga intervention. The blinded assessors reported that they were unaware of group allocation of any participant at follow up.

\section{Qualitative Satisfaction Questionnaire Responses}

Five themes regarding benefits of the yoga intervention emerged from the open-ended post-participation questionnaire responses: cognitive function, mental health, sleep, quality of life, and social interaction. Improved thinking was identified by six participants; one participant said, "[l'm] remembering pills, ... remembering to pick stuff up at the store". Five of nine participants in the yoga group reported stress relief or decreased depression as a benefit to the intervention. One participant said, "Doing the yoga sessions gave me new skills to deal with every day stresses and participating gave me something to look forward to weekly... I appreciate this study because it was something that had immediate results. It was something for 'me' and right from the beginning, it felt less of a research project and more of a therapy course." Four participants identified improved quality of life, with two specifically reporting enhanced energy as a result of the intervention. Three participants identified social interaction as a benefit of yoga as exemplified by a participant who said, "[l've] noticed that my behavior and attitude toward others has improved by the help of yoga". Others identified enhanced sleep as a result of the intervention. A participant noted, "I would sleep 4-5 hours before the yoga classes, now I can sleep 7-8 hours after the classes". One participant noted that the virtual classes were helpful, "[The] instructor was fantastic about accommodating my schedule. However, I just wish I could have attended more classes".

\section{Descriptive statistics}


There were no missing data for the second, third, or fourth outcomes. B-CAM, comfortable and fast gait speed, and MOS-HIV general health, mental health, and energy subscales met the assumptions for parametric tests while the other outcomes did not. Mean B- CAM scores in the overall sample at baseline were 19.0 \pm 4.6 and 19.6 \pm 5.3 at study completion. The mean C3Q scores of the overall sample were $24.6 \pm 8.1$ at baseline and $25.7 \pm 6.5$ at 12 weeks. Mean overall CB\&M scores at baseline were $67.1 \pm 22.3$ and $68.7 \pm 22.5$ at 12 weeks. The mean score on the CB\&M in the overall sample (including baseline and final scores) was $67.9( \pm 21.1$, range $=22-96)$. One participant $(2.38 \%$ out of 42 observations) obtained a maximum score of 96 on the CB\&M, and none of the participants obtained the minimum score of 0 .

\section{Effect of the intervention}

Results of the intention-to-treat analyses $(n=22)$ are reported. When we re-analyzed the data using per protocol analyses, we found no differences in the results unless otherwise stated. We found no significant effect of gender between groups on all outcomes.

\section{Cognitive performance (second objective)}

A summary of results for cognitive performance is shown in Table 2. There were no significant between-group effects of the yoga intervention on the B-CAM scores at study completion $[F(1,16)=.687, C l(-1.164,5.718) p$ $=.353]$. There were also no within-group differences on the B-CAM scores over time, as determined by the mixed ANCOVA $[F(1,17)=1.769, \mathrm{Cl}(-2.979, .746), p=.201]$. Self-reported cognition (C3Q) scores did not differ significantly between the groups at study completion $[F(1,16)=.002, \mathrm{Cl}(-8.362,8.563), p=.965]$.. There were also no significant within-group differences in C3Q scores (yoga group $Z=-.983, p=.326$; control group $Z=-.089, p=.929$ ).

Table 2: Cognitive outcomes

\begin{tabular}{|c|c|c|c|c|c|c|c|c|c|c|c|}
\hline Outcome & $\begin{array}{l}\text { Yoga } \\
\text { baseline } \\
\text { mean } \\
(\mathrm{SD})\end{array}$ & $\begin{array}{l}\text { Yoga } \\
\text { post } \\
\text { mean } \\
\text { (SD) } \\
\end{array}$ & $\begin{array}{l}\text { Yoga } \\
\text { mean } \\
\text { change } \\
\text { (SD) } \\
\end{array}$ & $\begin{array}{l}\text { Control } \\
\text { baseline } \\
\text { (SD) }\end{array}$ & $\begin{array}{l}\text { Control } \\
\text { post } \\
\text { (SD) }\end{array}$ & $\begin{array}{l}\text { Control } \\
\text { mean } \\
\text { change } \\
(\mathrm{SD}) \\
\end{array}$ & $\begin{array}{l}95 \% \\
\text { confidence } \\
\text { interval }\end{array}$ & $\begin{array}{l}\text { P value } \\
\text { (within } \\
\text { groups) }\end{array}$ & $\begin{array}{l}\text { P value } \\
\text { (between } \\
\text { groups) }\end{array}$ & $\begin{array}{l}\text { Gender } \\
\text { analysis } \\
\text { (p- } \\
\text { value) } \\
\end{array}$ & Effect size \\
\hline B-CAM & $\begin{array}{l}22.07 \\
(3.51)\end{array}$ & $\begin{array}{l}22.27 \\
(4.38)\end{array}$ & $\begin{array}{l}0.36 \\
(3.55)\end{array}$ & $\begin{array}{l}16.26 \\
(3.53)\end{array}$ & $\begin{array}{l}18.22 \\
(5.69)\end{array}$ & $\begin{array}{l}1.97 \\
(4.29)\end{array}$ & $\begin{array}{l}{[-1.164} \\
5.718] \dagger\end{array}$ & .201 & $.353 \dagger$ & .325 & $\begin{array}{l}\text { Between: } \quad \eta p \square \\
=.041 \text { Within: } \\
\eta p \llbracket=.094\end{array}$ \\
\hline C3Q & $\begin{array}{l}26.09 \\
(8.03)\end{array}$ & $\begin{array}{l}28.11 \\
(5.49)\end{array}$ & $\begin{array}{l}1.78 \\
(5.14)\end{array}$ & $\begin{array}{l}23.18 \\
(8.36)\end{array}$ & $\begin{array}{l}25.45 \\
(7.24)\end{array}$ & $\begin{array}{l}2.27 \\
(11.19)\end{array}$ & $\begin{array}{l}{[-8.362,} \\
8.563] \dagger\end{array}$ & $\begin{array}{l}.375 \\
\text { (yoga) } \\
.948 \\
\text { (control) }\end{array}$ & $.965 \dagger$ & .760 & $\begin{array}{l}\text { Between: } \quad \eta p \square \\
=.000 \text { Within: } \\
\mathrm{r}=-.22 \\
\text { (yoga); } \\
\text {-.019 } \\
\text { (control) }\end{array}$ \\
\hline
\end{tabular}

†Bootstrap values are bias corrected and accelerated based on 1000 bootstrap replications. Effect sizes are partial eta squared ( $\eta p \rrbracket)$ values between groups

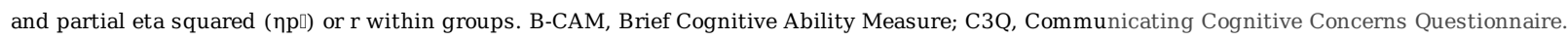

\section{Third objective outcomes}

Physical performance and physical activity outcomes

Physical performance and physical activity results are shown in Table 3. There were no significant within- or between-group differences in balance or gait speed. We did not observe between-group or within-group differences in 
physical activity as measured by the RAPA and accelerometer data.

Table 3: Physical performance and activity levels

\begin{tabular}{|c|c|c|c|c|c|c|c|c|c|c|c|}
\hline Outcome & $\begin{array}{l}\text { Yoga } \\
\text { baseline } \\
\text { mean } \\
\text { (SD) }\end{array}$ & $\begin{array}{l}\text { Yoga } \\
\text { post } \\
\text { mean } \\
\text { (SD) }\end{array}$ & $\begin{array}{l}\text { Yoga } \\
\text { mean } \\
\text { change } \\
\text { (SD) }\end{array}$ & $\begin{array}{l}\text { Control } \\
\text { baseline } \\
\text { (SD) }\end{array}$ & $\begin{array}{l}\text { Control } \\
\text { post } \\
\text { (SD) }\end{array}$ & $\begin{array}{l}\text { Control } \\
\text { mean } \\
\text { change } \\
\text { (SD) }\end{array}$ & $\begin{array}{l}95 \% \\
\text { confidence } \\
\text { interval }\end{array}$ & $\begin{array}{l}\text { P value } \\
\text { (within } \\
\text { groups) }\end{array}$ & $\begin{array}{l}\text { P value } \\
\text { (between groups) }\end{array}$ & $\begin{array}{l}\text { Gender } \\
\text { analysis } \\
\text { (p- } \\
\text { value) }\end{array}$ & Effect size \\
\hline $\mathrm{CB} \& \mathrm{M}$ & $\begin{array}{l}74.73 \\
(20.97)\end{array}$ & $\begin{array}{l}73.67 \\
(27.05)\end{array}$ & $\begin{array}{l}1.22 \\
(7.46)\end{array}$ & $\begin{array}{l}59.45 \\
(17.15)\end{array}$ & $\begin{array}{l}64.64 \\
(18.26)\end{array}$ & $\begin{array}{l}5.18 \\
(9.91)\end{array}$ & $\begin{array}{l}{[-2.011,} \\
36.723] \dagger\end{array}$ & $\begin{array}{l}.578 \\
\text { (yoga) } \\
.085 \\
\text { (control) }\end{array}$ & $.107 \dagger$ & .533 & $\begin{array}{l}\text { Between: } \\
\eta p \square \\
=.211 \\
\text { Within: } \\
\mathrm{r}=-.151 \text { (yoga); } \\
-.371 \text { (control) }\end{array}$ \\
\hline $\begin{array}{l}\text { Comfortable } \\
\text { gait speed }\end{array}$ & $\begin{array}{l}1.29 \\
(0.29)\end{array}$ & $\begin{array}{l}1.32 \\
(0.36)\end{array}$ & $\begin{array}{l}0.06 \\
(0.13)\end{array}$ & $\begin{array}{l}1.11 \\
(.26)\end{array}$ & $\begin{array}{l}1.20 \\
(0.35)\end{array}$ & $\begin{array}{l}0.09 \\
(0.17)\end{array}$ & {$[-.279, .481]$} & .876 & .581 & .338 & $\begin{array}{l}\text { Between: } \\
\eta p \square \quad= \\
.019 \\
\text { Within: } \\
\eta p \square=.002 \\
\end{array}$ \\
\hline $\begin{array}{l}\text { Fast gait } \\
\text { speed }\end{array}$ & $\begin{array}{l}1.79 \\
(0.40)\end{array}$ & $\begin{array}{l}1.73 \\
(0.48)\end{array}$ & $\begin{array}{l}-0.05 \\
(0.15)\end{array}$ & $\begin{array}{l}1.59 \\
(0.35)\end{array}$ & $\begin{array}{l}1.70 \\
(0.43)\end{array}$ & $\begin{array}{l}0.10 \\
(0.30)\end{array}$ & {$[-.221, .725]$} & .919 & .276 & .291 & $\begin{array}{l}\text { Between: } \\
\eta p \square \\
=\quad .074 \\
\text { Within: } \\
\eta p \square=.001\end{array}$ \\
\hline RAPA 1 & $\begin{array}{l}4.40 \\
(1.58)\end{array}$ & $\begin{array}{l}5.33 \\
(1.58)\end{array}$ & $\begin{array}{l}0.78 \\
(2.33)\end{array}$ & $\begin{array}{l}5.09 \\
(1.64)\end{array}$ & $\begin{array}{l}5.27 \\
(1.68)\end{array}$ & $\begin{array}{l}0.18 \\
(1.72)\end{array}$ & $\begin{array}{l}-2.716, \\
.797] \dagger\end{array}$ & $\begin{array}{l}.563 \\
\text { (yoga) } \\
.594 \\
\text { (control) }\end{array}$ & $.351 \dagger$ & .425 & $\begin{array}{l}\text { Between: } \\
\eta p \square \\
=.046 \\
\text { Within: } \\
\mathrm{r}=.192 \text { (yoga) } \\
\mathrm{r}=.150 \text { (control) }\end{array}$ \\
\hline RAPA 2 & $\begin{array}{c}1.20 \\
(1.32)\end{array}$ & $\begin{array}{l}2.44 \\
(0.53)\end{array}$ & $\begin{array}{l}1.11 \\
(1.62)\end{array}$ & $\begin{array}{l}1.09 \\
(1.38)\end{array}$ & $\begin{array}{l}1.09 \\
(1.30)\end{array}$ & $\begin{array}{l}.00 \\
(0.77)\end{array}$ & $\begin{array}{l}{[-1.892,} \\
.505] \dagger\end{array}$ & $\begin{array}{l}.125 \\
\text { (yoga) } \\
1.00 \\
\text { (control) }\end{array}$ & $.233+$ & .422 & $\begin{array}{l}\text { Between: } \\
\eta p \square \\
=.095 \\
\text { Within: } \\
\mathrm{r}=.42 \text { (yoga) } \\
\mathrm{r}=.00 \text { (control) }\end{array}$ \\
\hline Steps per day & $\begin{array}{l}9111.61 \\
(4147.03)\end{array}$ & $\begin{array}{l}8765.60 \\
(4697.4 \\
8)\end{array}$ & $\begin{array}{l}-24.06 \\
(1836.0 \\
9)\end{array}$ & $\begin{array}{l}6955.52 \\
(4937.7 \\
9)\end{array}$ & $\begin{array}{l}6155.56 \\
(3900.0 \\
2)\end{array}$ & $\begin{array}{l}-699.96 \\
(2221.3 \\
0)\end{array}$ & $\begin{array}{l}{[-1610.19,} \\
4074.68] \dagger\end{array}$ & $\begin{array}{l}1.00 \\
\text { (yoga) } \\
.374 \\
\text { (control) }\end{array}$ & $.431 \dagger$ & .957 & $\begin{array}{l}\text { Between: } \\
\eta p \square \\
=\quad .023 \\
\text { Within: } \\
\mathrm{r}=.014 \\
\text { (yoga) } \mathrm{r}= \\
.19 \text { (control) }\end{array}$ \\
\hline $\begin{array}{l}\text { Kilometers per } \\
\text { day }\end{array}$ & $\begin{array}{c}6.38 \\
(2.56)\end{array}$ & $\begin{array}{l}6.03 \\
(2.99)\end{array}$ & $\begin{array}{l}-0.05 \\
(1.45)\end{array}$ & $\begin{array}{l}4.83 \\
(3.45)\end{array}$ & $\begin{array}{l}4.33 \\
(2.62)\end{array}$ & $\begin{array}{l}-0.50 \\
(1.63)\end{array}$ & $\begin{array}{l}{[-2.837,} \\
2.887] \dagger\end{array}$ & $\begin{array}{l}1.00 \\
\text { (yoga) } \\
.374 \\
\text { (control) }\end{array}$ & $.834 \dagger$ & .834 & $\begin{array}{l}\text { Between: } \\
\eta p \square \\
=.002 \\
\text { Within: } \\
\mathrm{r}=.014 \text { (yoga) } \\
\mathrm{r}=.19 \text { (control) }\end{array}$ \\
\hline
\end{tabular}

†Bootstrap values are bias corrected and accelerated based on 1000 bootstrap replications. Effect sizes are partial eta squared ( $\eta p \rrbracket$ ) values between groups and partial eta squared ( $\eta p \rrbracket)$ or $r$ within groups. CB\&M, Community Balance and Mobility Scale; RAPA, Rapid Assessment of Physical Activity.

\section{Health-related quality of life, mental health, and antiretroviral adherence outcomes}

Health-related quality of life and mental health results are displayed in Table 4. There were significant within-group differences on the MOS-HIV cognitive subscale in the yoga group $(Z=-2.120, p=.047)$ only. We also observed within-group difference trends among yoga participants on the MOS-HIV health transition $(p=.063)$ and the HADSdepression ( $p=.055$ ) subscales. In contrast with the intention-to-treat analyses, per protocol analyses showed a trend in improved cognitive subscale scores $(p=.094)$ and no trend in improved health transition scores among yoga participants $(p=0.125)$. We did not observe between-group differences on any other MOS-HIV subscales or the HADS questionnaire. There was no effect of the intervention on antiretroviral adherence at study completion using the SMAQ (Chi-square $=1.650, p=.362)$. 
Table 4: Health-related quality of life and mental health outcomes

\begin{tabular}{|c|c|c|c|c|c|c|c|c|c|c|c|}
\hline Outcome & $\begin{array}{l}\text { Yoga } \\
\text { baseline } \\
\text { mean } \\
(\mathrm{SD}) \\
\end{array}$ & $\begin{array}{l}\text { Yoga } \\
\text { post } \\
\text { mean } \\
(\mathrm{SD}) \\
\end{array}$ & $\begin{array}{l}\text { Yoga } \\
\text { mean } \\
\text { change } \\
\text { (SD) }\end{array}$ & $\begin{array}{l}\text { Control } \\
\text { baseline } \\
\text { (SD) }\end{array}$ & $\begin{array}{l}\text { Control } \\
\text { post } \\
\text { (SD) }\end{array}$ & $\begin{array}{l}\text { Control } \\
\text { mean } \\
\text { change } \\
\text { (SD) }\end{array}$ & $\begin{array}{l}95 \% \\
\text { confidence } \\
\text { interval }\end{array}$ & $\begin{array}{l}\text { P value } \\
\text { (within } \\
\text { groups) }\end{array}$ & $\begin{array}{l}\text { P value } \\
\text { (between } \\
\text { groups) }\end{array}$ & $\begin{array}{l}\text { Gender } \\
\text { analysis } \\
\text { (p- value) }\end{array}$ & Effect size \\
\hline $\begin{array}{l}\text { General } \\
\text { Health }\end{array}$ & $\begin{array}{l}61.36 \\
(32.41)\end{array}$ & $\begin{array}{l}62.78 \\
(31.63)\end{array}$ & $\begin{array}{l}7.78 \\
(14.60)\end{array}$ & $\begin{array}{l}55.00 \\
(28.90)\end{array}$ & $\begin{array}{l}54.55 \\
(30.78)\end{array}$ & $\begin{array}{l}-0.45 \\
(19.68)\end{array}$ & $\begin{array}{l}{[-34.055} \\
34.011]\end{array}$ & .884 & .999 & .627 & $\begin{array}{l}\text { Between: } \\
\eta p[ \\
=\quad .000 \\
\text { Within: } \\
\eta p[=.001\end{array}$ \\
\hline Physical & $\begin{array}{l}81.82 \\
(25.23)\end{array}$ & $\begin{array}{l}82.41 \\
(24.10)\end{array}$ & $\begin{array}{l}3.70 \\
(13.89)\end{array}$ & $\begin{array}{l}68.94 \\
(25.30)\end{array}$ & $\begin{array}{l}76.51 \\
(17.41)\end{array}$ & $\begin{array}{l}7.58 \\
(21.56)\end{array}$ & $\begin{array}{c}{[-21.085} \\
40.728] \dagger\end{array}$ & $\begin{array}{l}.563 \\
\text { (yoga) } \\
.250 \\
\text { (control) } \\
\end{array}$ & $.545 \dagger$ & .799 & $\begin{array}{l}\text { Between: } \eta p[ \\
=.042 \text { Within: } \\
\mathrm{r}=-.19 \text { (yoga); } \\
-.25 \text { (control) }\end{array}$ \\
\hline Role & $\begin{array}{l}59.09 \\
(43.69)\end{array}$ & $\begin{array}{l}66.67 \\
(35.36)\end{array}$ & $\begin{array}{l}16.67 \\
(25.0)\end{array}$ & $\begin{array}{l}77.27 \\
(41.01)\end{array}$ & $\begin{array}{l}68.18 \\
(46.22)\end{array}$ & $\begin{array}{l}-9.09 \\
(37.54)\end{array}$ & $\begin{array}{l}{[-45.927} \\
44.243] \dagger\end{array}$ & $\begin{array}{l}.250 \\
\text { (yoga) } \\
.750 \\
\text { (control) } \\
\end{array}$ & $.890 \dagger$ & .621 & $\begin{array}{l}\text { Between: } \eta p \square \\
=.001 \text { Within: } \\
\mathrm{r}=-.41 \text { (yoga); -.17 } \\
\text { (control) }\end{array}$ \\
\hline Social & $\begin{array}{l}92.73 \\
(13.48)\end{array}$ & $\begin{array}{l}95.56 \\
(8.82)\end{array}$ & $\begin{array}{l}4.44 \\
(19.44)\end{array}$ & $\begin{array}{l}80.0 \\
(28.28)\end{array}$ & $\begin{array}{l}78.18 \\
(24.42)\end{array}$ & $\begin{array}{l}-1.82 \\
(27.50)\end{array}$ & $\begin{array}{l}{[.-32.297} \\
10.736] \dagger\end{array}$ & $\begin{array}{l}.750 \\
\text { (yoga) } \\
1.00 \\
\text { (control) } \\
\end{array}$ & $.536 \dagger$ & .326 & $\begin{array}{l}\text { Between: } \eta p \square \\
=.019 \text { Within: } \\
\mathrm{r}=-.17 \text { (yoga); }-.003 \\
\text { (control) }\end{array}$ \\
\hline Cognitive & $\begin{array}{l}70.00 \\
(19.11)\end{array}$ & $\begin{array}{l}78.89 \\
(17.82)\end{array}$ & $\begin{array}{l}10.56 \\
(12.86)\end{array}$ & $\begin{array}{l}75.45 \\
(14.57)\end{array}$ & $\begin{array}{l}84.09 \\
(17.44)\end{array}$ & $\begin{array}{l}8.63 \\
(18.18)\end{array}$ & $\begin{array}{l}{[-19.927} \\
36.067] \dagger\end{array}$ & $\begin{array}{l}.047^{*} \\
\text { (yoga) } \\
.191 \\
\text { (control) } \\
\end{array}$ & $.475 \dagger$ & .923 & $\begin{array}{l}\text { Between: } \eta p \llbracket=.046 \\
\text { Within: } \\
\mathrm{r}=-.52 \text { (yoga); }-.296 \\
\text { (control) }\end{array}$ \\
\hline Pain & $\begin{array}{l}76.77 \\
(19.53)\end{array}$ & $\begin{array}{l}(72.84 \\
(23.64)\end{array}$ & $\begin{array}{l}-3.70 \\
(20.03)\end{array}$ & $\begin{array}{l}60.61 \\
(25.03)\end{array}$ & $\begin{array}{l}53.54 \\
(28.90)\end{array}$ & $\begin{array}{l}-7.07 \\
(17.41)\end{array}$ & $\begin{array}{l}{[-47.781} \\
10.821] \dagger\end{array}$ & $\begin{array}{l}.813 \\
\text { (yoga) } \\
.305 \\
\text { (control) } \\
\end{array}$ & $.323 \dagger$ & .570 & $\begin{array}{l}\text { Between: } \eta \mathrm{p} \square=.089 \\
\text { Within: } \\
\mathrm{r}=-.10 \text { (yoga); } \\
-.26 \text { (control) }\end{array}$ \\
\hline Mental & $\begin{array}{l}74.91 \\
(17.63)\end{array}$ & $\begin{array}{l}80.00 \\
(17.78)\end{array}$ & $\begin{array}{l}6.67 \\
(13.71)\end{array}$ & $\begin{array}{l}77.82 \\
(17.38)\end{array}$ & $\begin{array}{l}70.18 \\
(19.46)\end{array}$ & $\begin{array}{l}-7.63 \\
(16.82)\end{array}$ & $\begin{array}{l}{[-30.372,} \\
6.786]\end{array}$ & .112 & .197 & .980 & $\begin{array}{l}\text { Between: } \\
\eta p \square \\
=\quad .102 \\
\text { Within: } \\
\eta p \square=.150 \\
\end{array}$ \\
\hline Energy & $\begin{array}{l}64.09 \\
(16.86)\end{array}$ & $\begin{array}{l}69.44 \\
(17.756)\end{array}$ & $\begin{array}{l}8.33 \\
(12.50)\end{array}$ & $\begin{array}{l}61.36 \\
(19.25)\end{array}$ & $\begin{array}{l}56.36 \\
(24.71)\end{array}$ & $\begin{array}{l}-5.0 \\
(18.30)\end{array}$ & $\begin{array}{l}{[-31.122} \\
17.215]\end{array}$ & .205 & .550 & .555 & $\begin{array}{l}\text { Between: } \\
\eta p \square \\
=\quad .023 \\
\text { Within: } \\
\eta p \square=.098\end{array}$ \\
\hline $\begin{array}{l}\text { Health } \\
\text { Distress }\end{array}$ & $\begin{array}{l}79.55 \\
(21.27)\end{array}$ & $\begin{array}{l}69.44 \\
(24.30)\end{array}$ & $\begin{array}{l}6.67 \\
(19.20)\end{array}$ & $\begin{array}{l}80.91 \\
(17.00)\end{array}$ & $\begin{array}{l}70.45 \\
(29.19)\end{array}$ & $\begin{array}{l}-1.82 \\
(25.52)\end{array}$ & $\begin{array}{l}{[-24.460} \\
20.790] \dagger\end{array}$ & $\begin{array}{l}.313 \\
\text { (yoga) } \\
.977 \\
\text { (control) }\end{array}$ & $.719 \dagger$ & .399 & $\begin{array}{l}\text { Between: } \eta p \square \\
=.005 \text { Within: } \\
r=-.27 \text { (yoga); -.01 } \\
\text { (control) }\end{array}$ \\
\hline $\begin{array}{l}\text { Quality of } \\
\text { life }\end{array}$ & $\begin{array}{l}75.0 \\
(22.36)\end{array}$ & $\begin{array}{l}77.78 \\
(19.54)\end{array}$ & $\begin{array}{l}8.33 \\
(12.50)\end{array}$ & $\begin{array}{l}70.45 \\
(21.85)\end{array}$ & $\begin{array}{l}68.18 \\
(19.66)\end{array}$ & $\begin{array}{l}-2.27 \\
(26.11)\end{array}$ & $\begin{array}{l}{[-27.696,} \\
17.203] \dagger\end{array}$ & $\begin{array}{l}.250 \\
\text { (yoga) } \\
.625 \\
\text { (control) } \\
\end{array}$ & $.535 \dagger$ & .792 & $\begin{array}{l}\text { Between: } \eta p \square \\
=.020 \text { Within: } \\
\mathrm{r}=-.41 \text { (yoga); }-.09 \\
\text { (control) }\end{array}$ \\
\hline $\begin{array}{l}\text { Health } \\
\text { Transition }\end{array}$ & $\begin{array}{l}56.82 \\
(19.66)\end{array}$ & $\begin{array}{l}69.44 \\
(26.41)\end{array}$ & $\begin{array}{l}19.44 \\
(20.83)\end{array}$ & $\begin{array}{l}56.82 \\
(22.61)\end{array}$ & $\begin{array}{l}70.45 \\
(29.19)\end{array}$ & $\begin{array}{l}13.63 \\
(30.34)\end{array}$ & $\begin{array}{l}{[-33.667} \\
50.265\end{array}$ & $\begin{array}{l}.063^{\wedge} \\
\text { (yoga) } \\
.176 \\
\text { (control) }\end{array}$ & $.714 \dagger$ & .681 & $\begin{array}{l}\text { Between: } \eta p \square \\
=.013 \text { Within: } \\
r=-.49 \text { (yoga); -.31 } \\
\text { (control) }\end{array}$ \\
\hline HADS-A & $\begin{array}{l}6.91 \\
(4.16)\end{array}$ & $\begin{array}{l}6.00 \\
(3.35)\end{array}$ & $\begin{array}{l}-1.33 \\
(2.69)\end{array}$ & $\begin{array}{l}7.09 \\
(4.01)\end{array}$ & $\begin{array}{l}5.36 \\
(2.66)\end{array}$ & $\begin{array}{l}-1.73 \\
(3.47)\end{array}$ & $\begin{array}{l}{[-3.776-} \\
3.329] \dagger\end{array}$ & $\begin{array}{l}.180 \\
\text { (yoga) } \\
.219 \\
\text { (control) } \\
\end{array}$ & $.893 \dagger$ & .678 & $\begin{array}{l}\text { Between: } \eta \mathrm{p} \square= \\
.000 \text { Within: } \\
\mathrm{r}=-.360 \text { (yoga); -.285 } \\
\text { (control) }\end{array}$ \\
\hline HADS-D & $\begin{array}{l}3.27 \\
(3.19)\end{array}$ & $\begin{array}{l}2.11 \\
(1.61)\end{array}$ & $\begin{array}{l}-1.44 \\
(2.24)\end{array}$ & $\begin{array}{l}5.27 \\
(4.00)\end{array}$ & $\begin{array}{l}4.27 \\
(3.92)\end{array}$ & $\begin{array}{l}-1.0 \\
(3.46)\end{array}$ & $\begin{array}{l}{[-1.460} \\
7.522] \dagger\end{array}$ & $\begin{array}{l}.055^{\wedge} \\
\text { (yoga) } \\
.363 \\
\text { (control) }\end{array}$ & $.147 \dagger$ & .272 & $\begin{array}{l}\text { Between: } \eta \mathrm{p} \square=.149 \\
\text { Within: } \\
\mathrm{r}=-.475 \text { (yoga); } \\
-.211 \text { (control) }\end{array}$ \\
\hline
\end{tabular}

†Bootstrap values are bias corrected and accelerated based on 1000 bootstrap replications. Effect sizes are partial eta squared ( $\eta p \rrbracket$ ) values between groups and partial eta squared $(\eta p \sqcap)$ or $r$ within groups. ${ }^{*} p<0.05 . \wedge p<0.10$ (trend).

\section{Supplementary analyses (fourth objective)}




\section{Associations with cognitive performance}

We created a correlation matrix to determine the relationship between self-reported cognition (C3Q) and cognitive performance (B-CAM) scores, revealing that baseline C3Q was not associated with B-CAM scores at baseline $[r=.262$, $p=.239]$. At study completion, however, C3Q was significantly moderately associated with B-CAM scores $[r=.585, p$ $=.007]$. Baseline balance (CB\&M) scores were strongly associated with cognitive performance (B-CAM) scores at baseline $[r=.843, p=.000]$ and final balance scores were associated with post- intervention B-CAM scores $[r=.612, p$ $=.004$ ]. Physical activity was also related to cognitive performance in this sample. The number of steps at baseline was related cognitive performance (B-CAM) at baseline $[r=.685, p=.000]$ and study completion $[r=.534, p=.015]$.

Furthermore, baseline kilometers walked per day was associated with both baseline $[r=.660, p=.001]$ and final BCAM scores $[r=.504, p=.023]$.

\section{Moderator analysis}

We found a significant interaction between CB\&M and B-CAM scores at baseline in the prediction of B-CAM scores at follow up in the yoga group $[B-0.49, \mathrm{Cl}(-.089,-.009), \mathrm{p}=0.026]$ but not the control group $[B 0.002, \mathrm{Cl}(-.046, .050), p=$ 0.914].

\section{Power calculation for future trial}

We performed a power analysis for cognitive performance (B-CAM) to determine the sample size for a future, fullscale randomized controlled trial. The sample size for this effect size $\left(n p^{2}=0.041\right)$ assuming $80 \%$ power and alpha of 0.05 , is 48 participants. We can expect a drop-out rate of $\sim 15 \%$, therefore the sample size for future work should include at least 55 participants.

\section{Discussion}

\section{Feasibility}

This RCT was the first to evaluate the feasibility and impact of a 12-week yoga intervention on cognitive and physical outcomes among a sample of PLWH. The intervention was feasible as determined by reasonable assessment times, lack of known adverse events, adherence to the intervention, low attrition, and participant satisfaction. No known injuries occurred as a result of the yoga intervention, providing evidence that this is a safe form of exercise for PLWH. Furthermore, the participants reported $100 \%$ satisfaction and comfort with the intervention. Although we did not achieve the anticipated sample size within the time frame to conduct the study, we met the a priori targets for adherence and satisfaction, which indicate that a future large-scale RCT should be conducted. In order to meet recruitment targets, future RCTs evaluating cognitive and physical performance outcomes among PLWH should use multiple sites. Our findings can inform future power calculations for large scale RCTs evaluating cognitive and physical outcomes among PLWH.

The rates of attrition (less than 10\%) and adherence to the yoga classes (82\%) in this study are encouraging and are likely due to high participant satisfaction. Three meta-analyses59- 61 and one systematic scoping review18 have identified high levels of withdrawal from exercise trials (20-29\%) and mind-body interventions (39\%), as well as variable adherence (61-100\%) among PLWH to these interventions. Another study evaluated the feasibility of a yoga 
intervention for PLWH and observed similar feasibility outcomes to our study, reporting an average of $88 \%$ attendance to the classes and $83 \%$ retention at study completion. 62 However, retention in the control group was poor (50\%) in the aforementioned study and the authors posited that this could be attributed to a lack of meaningful intervention. 62 In comparison, we had 100\% retention in our control group at study completion, likely due to the short study duration and small time commitment.

\section{Cognitive performance}

We found no significant improvement in cognitive performance following the 12-week yoga intervention. This finding is in keeping with those of McDermott et al. (2017), whose 16- week aerobic exercise intervention $(n=11)$ did not have an effect on cognitive performance among PLWH.63 Evidence from the cross-sectional literature has showed positive associations between physical activity and better cognitive function among PLWH,64-71 but RCT evidence has yet to determine a causal relationship. There are a few potential reasons for the lack of effect of the intervention on cognitive performance in the present study. Firstly, the small sample size was not sufficient to detect a change in outcomes. Secondly, there may have been some factors related to the dose of the intervention. For example, results from a meta-analysis of exercise moderators among older healthy adults indicates that more frequent (5-7 sessions per week) exercise sessions have larger benefits for cognitive performance than less frequent sessions (3-4 per week).72 The intervention may also have been too short in total duration, as evidenced by a systematic review that determined that total exercise duration was the most potent predictor of cognitive performance and the authors recommended that exercise interventions for older adults last at least 52 hours in total.73 Finally, the impact of social engagement on cognitive performance should be investigated further in the HIV population. Fazeli and colleagues (2014) performed a cross-sectional analysis and concluded that the combination of physical activity with social interaction and employment had a stronger impact on cognitive performance than each of these factors alone.64 Future large-scale RCTs evaluating cognitive outcomes among PLWH should involve longer exercise interventions and active control groups.

\section{Self-reported cognition}

Despite the lack of improvement in objective cognitive performance in this sample, there were significant improvements over time on the cognitive subscale of the MOS-HIV among yoga participants. This finding is surprising, as we did not observe concurrent improvements in self-reported cognition in the yoga group when measured using the $\mathrm{C} 3 \mathrm{Q}$. Both questionnaires have items measuring self-reported executive function, attention/concentration, and memory but the items on the $\mathrm{C} 3 \mathrm{Q}$ are more specific than those in the cognitive subscale of the MOS-HIV, and the C3Q also includes items on language and motivation.32 Self-reported cognition is an important clinical outcome in this population. As such, more research is needed to determine a gold-standard measure of self-reported cognition among PLWH.

We observed significant improvements over time among yoga participants on the MOS- HIV cognitive subscale with an intention-to-treat analysis but no improvement when using a per- protocol analysis. This is an unexpected result as intention-to-treat analyses are typically more conservative.74 Our results may be explained by a participant in the yoga group who did not achieve the $70 \%$ adherence target but had improvements in self-reported cognition.

\section{Health-related quality of life and mental health}


There were trends toward improvements in the MOS-HIV health transition subscale and depression as measured using the HADS among yoga participants. We also found qualitative reports of improved social interaction among yoga participants. This evidence is in keeping with other literature evaluating the effect of mind-body interventions on health-related quality of life outcomes among PLWH. An observational study of male and female PLWH determined that Tai Chi benefited cognitive health-related quality of life as measured by the FAHI (Functional Assessment of HIV Infection).21 Another study found a positive effect of a yogic breathing and meditation intervention on the general health, social function and cognitive function subscales of the MOS-HIV among PLWH.75 Galantino and colleagues (1997) observed within-group differences in their aerobic exercise and Tai Chi groups on health transition scores of the MOS- HIV, with no corresponding changes in the control group among men with AIDS.76 Similarly, a pilot RCT determined that a meditation intervention enhanced health-related quality of life in a sample of mostly male PLWH.77 In contrast, Agarwal et al. (2015) did not find an effect of yoga on health-related quality of life among mostly male PLWH as measured using the Short Form-36.62 However, the demographics between that study were different than the present study as their inclusion criteria were PLWH who used crack cocaine.

\section{Physical performance}

There is some RCT evidence to indicate that yoga can improve balance among older adults without HIV.78-81 In fact, it appears that yoga is as effective as Tai Chi and balance training at improving balance performance among older fallers.82 However, in the present study, we found no effect of yoga on dynamic balance. This lack of effect may be related to the variation in balance performance observed among some participants in the control group and the small sample size. No other studies have evaluated CB\&M performance among PLWH, but baseline values in this study were lower than normative values for healthy adults in all age categories except one (ages 60-69).83 To date, no balance measures have been validated with PLWH. Further research in this area is needed to investigate balance impairments and appropriate balance performance measures in this population.

\section{Association between cognitive and physical performance}

The strong association between cognitive performance and balance found in this study is of interest, as this finding corroborates the results of other studies conducted with healthy older adults and individuals with mild cognitive impairment. A study conducted with older adults from South Africa showed a negative relationship between time taken to tandem walk (where the toes of the back foot touch the heel of the front foot during each step) for 10 metres and the number of correct responses on the Stroop task, which is indicative of an association between physical and cognitive performance.84 Another study conducted with healthy older adults from Malaysia determined that a positive relationship exists between dynamic balance and digit symbol test and matrix reasoning tests. 85 In the same study, an agility test predicted performance on cognitive tests such as the digit span test, clock drawing test, and the Mini Mental State Examination.85 A study conducted with older adults with mild cognitive impairment determined that comfortable gait speed and Timed Up \& Go performance were each associated with executive function performance using the Trails Making B test and the Stroop-Interference test.86 Among individuals with subcortical dementia, a reduction in gait speed is related to changes in subcortical structures, connections between frontal and subcortical structures, and subcortical neurotransmitters. 87

There is evidence of an association between physical and cognitive performance among PLWH as well. A large multi-site study of 1,549 mostly male PLWH and HIV-negative individuals reported that slowed gait was strongly 
associated with worse neuropsychological test scores.13 Another study of 164 mostly female PLWH determined that motor impairment (as measured by the HIV Dementia Motor Scale), cognitive dysfunction, and cerebrovascular disease were related to each other at baseline.88 The underlying pathology of this relationship is thought to include inflammation and underlying cardiovascular comorbidities.88,89 Future work should evaluate the interaction between balance and cognitive performance among PLWH with a larger sample.

\section{Study limitations}

There are several limitations to the present study that may have contributed to our findings. The principal investigator taught the yoga classes to the participants, which could bias the results. Recruitment was limited by the small number of PLWH in the geographical area. We also were only able to recruit 5 PLWH who identified as female. Due to the small number of female and two-spirited participants, it was not possible for us to report the results disaggregated by gender. Rather, gender was entered into the analyses for each variable as a covariate to determine if differences existed between groups. As a result, sex and gender may have had an unknown effect on the uptake and impact of the yoga intervention. We also did not report study withdrawal by gender to avoid identifying participants. The small sample size may have negatively affected the process of randomization, as it is difficult to obtain equal groups 90 which may have had a confounding effect on outcomes. Changes in medications, substance use, and other factors could have contributed to the variation in cognitive and physical performance observed in this sample. As such, stratification based on cognitive performance at baseline may be useful for future RCTs. Finally, the use of a passive control group limited our ability to determine the effect of social interaction on cognitive and affective outcomes.

\section{Conclusion}

This 12-week yoga intervention was feasible as determined by assessment protocols, adherence to yoga sessions, small attrition rates, and participant satisfaction. Our results indicate that a future multi-site RCT is warranted. Future work should include an active control group and an intervention lasting at least $\mathbf{5 2}$ hours in total in order to investigate the effectiveness of yoga in this population. We found no significant differences in cognitive or physical performance between groups but there was a significant improvement in MOS-HIV cognitive subscale scores, and trends toward improvements in health-related quality of life subscales and depression scores in the yoga group following the intervention. Future research should follow the SAGER guidelines and employ sex and gender-based analyses in order to explore the impact of yoga interventions on health outcomes across different sexes and genders among PLWH.

\section{Declarations}

\section{Declaration of conflicting interests}

The authors declare that there is no conflict of interest.

\section{Funding acknowledgements}

This work was supported by a Canadian Institutes of Health Research Catalyst Grant in HIV/AIDS Community Based Research (371452) and a Physiotherapy Foundation of Canada Neurosciences Division Award for Physiotherapy Research. 


\section{References}

1. AIDS by the numbers. UNAIDS.

2. UNAIDS Data 2018. Program HIV/AIDS. 2018:1-376.

http://www.unaids.org/sites/default/files/media_asset/unaids-data-

2018_en.pdf\%0Ahttp://www.unaids.org/sites/default/files/media_asset/20170720_Data_b ook_2017_en.pdf.

3. Young People and HIV/AIDS. https://www.unaids.org/sites/default/files/media_asset/2019_women-andhiv_en.pdf.

4. Heaton RK, Clifford DB, Franklin DR, et al. HIV-associated neurocognitive disorders persist in the era of potent antiretroviral therapy: CHARTER Study. Neurology. 2010;75(23):2087. doi:10.1212/WNL.0b013e318200d727

5. Simioni S, Cavassini M, Annoni JM, et al. Cognitive dysfunction in HIV patients despite long-standing suppression of viremia. AIDS. 2010;24(9):1243-1250. doi:10.1097/QAD.0b013e3283354a7b

6. Sacktor N. Changing clinical phenotypes of HIV-associated neurocognitive Journal of NeuroVirology. 2017:1-5.

7. Bonnet F, Amieva H, Marquant F, et al. Cognitive disorders in HIV-infected Aids. 2013;27(3):391-400. doi:10.1097/qad.0b013e32835b1019

8. Goodkin K. Psychiatric Aspects of HIV Spectrum Disease. Focus (Madison). 2009;7(3):303-310.

9. Grant I, Heaton RK, Marcotte TD, et al. Neurocognitive disturbances in HIV. Int Rev Psychiatry. 2008;20(1):33-47. doi:10.1080/09540260701877894

10. Maki PM, Rubin LH, Springer G, et al. Differences in cognitive function between women and men with HIV. $J$ Acquir Immune Defic Syndr. 2018;79(1):101-107. doi:10.1097/QAI.0000000000001764

11. Bauer LO, Wu Z, Wolfson LI. An obese body mass increases the adverse effects of HIV/AIDS on balance and gait. Phys Ther. 2011;91(7):1063. doi:10.2522/ptj.20100292

12. Richert $L$, Brault $M$, Mercié $P$, et al. Decline in locomotor functions over time in HIV-infected patients. AIDS. 2014;28(10):1441-1449. doi:10.1097/QAD.0000000000000246

13. Robertson K, Parsons T, Sidtis J, et al. Timed Gait test: Normative data for the assessment of the AIDS dementia complex. J Clin Exp Neuropsychol. 2006;28(7):1053-1064. doi:10.1080/13803390500205684

14. Wu C, Yi Q, Zheng X, et al. Effects of Mind-Body Exercises on Cognitive Function in Older Adults: A MetaAnalysis. J Am Geriatr Soc. 2018;67(4):749-758. doi:10.1111/jgs.15714

15. Zhang Y, Li C, Zou L, Liu X, Song W. The Effects of Mind-Body Exercise on Cognitive Performance in Elderly: A Systematic Review and Meta-Analysis. Int J Environ Res Public Health. 2018;15(12).

doi:10.3390/ijerph15122791

16. Innes KE, Bourguignon C, Taylor AG. Risk Indices Associated with the Insulin Resistance Syndrome, Cardiovascular Disease, and Possible Protection with Yoga: A Systematic Review. J Am Board Fam Med. 2005;18(6):491-519. doi:10.3122/jabfm.18.6.491

17. Ross A, Thomas S. The health benefits of yoga and exercise: a review of comparison studies. J Altern Complement Med. 2010;16(1):3. doi:10.1089/acm.2009.0044

18. Ramirez-Garcia MP, Gagnon MP, Colson S, Côté J, Flores-Aranda J, Dupont M. Mind- body practices for people living with HIV: A systematic scoping review. BMC Complement Altern Med. 2019;19(1). doi:10.1186/s12906019-2502-z

19. Lorenc A, Robinson N. A Review of the Use of Complementary and Alternative Medicine and HIV: Issues for Patient Care. AIDS Patient Care STDs. 2013;27(9):503. doi:10.1089/apc.2013.0175 
20. Braun LA, Forrester CA, Rawlins MDM, et al. Complementary medicine use by people living with HIV in Australia - a national survey. Int J STD AIDS. 2016;27(1):33-38. doi:10.1177/0956462415573122

21. Robins JLW, McCain NL, Gray DP, K ER, Walter JM, McDade E. Research on psychoneuroimmunology: Tai Chi as a stress management approach for individuals with HIV disease. App/ Nurs Res. 2006;19(1):2-9

22. Galantino M Lou, Shepard K, Krafft L, et al. The effect of group aerobic exercise and T'ai chi on functional outcomes and quality of life for persons living with acquired immunodeficiency syndrome. $J$ Altern Complement Med. 2005;11(6):1085-1092. doi:10.1089/acm.2005.11.1085

23. Kuloor A, Kumari S, Metri K. Impact of yoga on psychopathologies and quality of life in persons with HIV: A randomized controlled study. J Bodyw Mov Ther. 2019;23(2):278- 283. doi:10.1016/j.jbmt.2018.10.005

24. Mawar N, Katendra T, Bagul R, et al. Sudarshan Kriya yoga improves quality of life in healthy people living with HIV (PLHIV): results from an open label randomized clinical trial. Indian J Med Res. 2015;141(1):90-99. doi:10.4103/0971-5916.154509

25. Naoroibam R, Metri KG, Bhargav H, Nagaratna R, Nagendra HR. Effect of Integrated Yoga (IY) on psychological states and CD4 counts of HIV-1 infected patients: A randomized controlled pilot study. Int J Yoga. 2016;9(1):57. doi:10.4103/0973-6131.171723

26. Dunne EM, Balletto BL, Donahue ML, et al. The benefits of yoga for people living with HIV/AIDS: A systematic review and meta-analysis. Complement Ther Clin Pract. 2019;34:157-164. doi:10.1016/j.ctcp.2018.11.009

27. Kietrys DM, Galantino M Lou, Cohen ET, et al. Yoga for persons with HIV-related distal sensory polyneuropathy: A case series. Rehabil Oncol. 2018;36(2):123-131. doi:10.1097/01.RE0.0000000000000089

28. Curno MJ, Rossi S, Hodges-Mameletzis I, Johnston R, Price MA, Heidari S. A Systematic Review of the Inclusion (or Exclusion) of Women in HIV Research: From Clinical Studies of Antiretrovirals and Vaccines to Cure Strategies. J Acquir Immune Defic Syndr. 2016;71(2):181-188. doi:10.1097/QAI.0000000000000842

29. Heidari S, Babor TF, De Castro P, Tort S, Curno M. Sex and Gender Equity in Research: rationale for the SAGER guidelines and recommended use. Heidari S, ed. Res Integr peer Rev. 2016;1(1):2. doi:10.1186/s41073-0160007-6

30. Eldridge SM, Chan CL, Campbell MJ, et al. CONSORT 2010 statement: extension to randomised pilot and feasibility trials. Pilot Feasibility Stud. 2016;2(1):64. doi:10.1186/s40814-016-0105-8

31. Moher D, Hopewell S, Schulz KF, et al. CONSORT 2010 explanation and elaboration: Updated guidelines for reporting parallel group randomised trials. J Clin Epidemiol. 2010;343. doi:10.1016/j.ijsu.2011.10.001

32. Askari S, Fellows L, Brouillette MJ, Moriello C, Duracinsky M, Mayo NE. Development of an item pool reflecting cognitive concerns expressed by people with HIV. Am J Occup Ther. 2018;72(2):1-9. doi:10.5014/ajot.2018.023945

33. Bredin SSD, Gledhill N, Jamnik VK, Warburton DER. PAR-Q+ and ePARmed-X+: new risk stratification and physical activity clearance strategy for physicians and patients alike. Can Fam Physician. 2013;59(3):273-277.

34. Quigley A, O’Brien KK, Brouillette M-J, MacKay-Lyons M. Evaluating the Feasibility and Impact of a Yoga Intervention on Cognition, Physical Function, Physical Activity, and Affective Outcomes in People Living With HIV: Protocol for a Randomized Pilot Trial. JMIR Res Protoc. 2019;8(5). doi:10.2196/13818

35. Uebelacker LA, Epstein-Lubow G, Gaudiano BA, Tremont G, Battle CL, Miller IW. Hatha yoga for depression: Critical review of the evidence for efficacy, plausible mechanisms of action, and directions for future research. $J$ Psychiatr Pract. 2010;16(1):22-33. doi:10.1097/01.pra.0000367775.88388.96

36. Cohen ET, Kietrys D, Fogerite SG, et al. Feasibility and impact of an 8-week integrative yoga program in people with moderate multiple sclerosis- related disability: A pilot study. Int J MS Care. 2017;19(1):30-39. 
doi:10.7224/1537-2073.2015-046

37. Brems $\mathrm{C}$, Colgan D, Freeman $\mathrm{H}$, et al. Elements of yogic practice: Perceptions of students in healthcare programs. Int J Yoga. 2016;9(2):121-129. doi:10.4103/0973-6131.183710

38. Morgan R, Freeman L. The Healing of Our People: Substance Abuse and Historical Trauma. Subst Use Misuse. 2009;44(1):84-98. doi:10.1080/10826080802525678

39. Roos R, Myezwa H, van Aswegen H. "Not easy at all but I am trying": Barriers and facilitators to physical activity in a South African cohort of people living with HIV participating in a home-based pedometer walking programme. AIDS Care. 2015;27(2):235. doi:10.1080/09540121.2014.951309

40. Mayo NE, Brouillette M-J, Fellows LK. Understanding and optimizing brain health in HIV now: protocol for a longitudinal cohort study with multiple randomized controlled trials. BMC Neurol. 2016;16(1):8. doi:10.1186/s12883-016-0527-1

41. Koski L, Brouillette $M$, Lalonde $\mathrm{R}$, et al. Computerized testing augments pencil-and- paper tasks in measuring HIV- associated mild cognitive impairment. HIV Med. 2011;12(8):472-480. doi:10.1111/j.14681293.2010.00910.x

42. Knorr S, Brouwer B, Garland SJ. Validity of the Community Balance and Mobility Scale in Community-Dwelling Persons After Stroke. Arch Phys Med Rehabil. 2010;91(6):890- 896. doi:10.1016/j.apmr.2010.02.010

43. Studenski SA, Perera S, Patel K, et al. Gait speed and survival in older adults. JAMA- Journal Am Med Assoc. 2011;305:50-58. doi:10.1001/jama.2010.1923

44. Topolski TD, LoGerfo J, Patrick DL, Williams B, Walwick J, Patrick MAJMB. The Rapid Assessment of Physical Activity (RAPA) Among Older Adults. Prev Chronic Dis. 2006;3(4).

45. Fitbit Flex 2TM Fitness Wristband. 2018. https://www.fitbit.com/au/flex2. Accessed November 29, 2018.

46. Knobel $\mathrm{H}$, Alonso J, Casado JL, et al. Validation of a simplied medication adherence questionnaire in a large cohort of HIV-infected patients : the GEEMA Study. AIDS. 2002;16(4):605-613.

47. Skevington SM, O'Connell KA. Measuring Quality of Life in HIV and AIDS: A Review of the Recent Literature. Psychol Health. 2003;18(3):331-350. doi:10.1080/0887044031000084030

48. Zigmond AS, Snaith RP. The Hospital Anxiety and Depression Scale. Acta Psychiatr Scand. 1983;67(6):361-370. doi:10.1111/j.1600-0447.1983.tb09716.x

49. Software - REDCap. REDCap - Research Electronic Data Capture. https://www.project-redcap.org/software/. Published 2017. Accessed August 18, 2018.

50. Braun V, Clarke V. Using thematic analysis in psychology. Qual Res Psychol. 2006;3(2):77-101. doi:10.1191/1478088706qp063oa

51. Akoglu H. User's guide to correlation coefficients. Turkish J Emerg Med. 2018;18(3):91- 93. doi:10.1016/j.tjem.2018.08.001

52. Henderson AR. The bootstrap: A technique for data-driven statistics. Using computer- intensive analyses to explore experimental data. Clin Chim Acta. 2005;359(1):1-26. doi:10.1016/j.cccn.2005.04.002

53. Walters SJ, Campbell MJ. The use of bootstrap methods for analysing health-related quality of life outcomes (particularly the SF-36). Health Qual Life Outcomes. 2004;2:70. doi:10.1186/1477-7525-2-70

54. SPSS Statistics. https://www.ibm.com/support/knowledgecenter/SSLVMB_25.0.0/statistics_kc_ddita/spss/ product_landing.html. Published 2017. Accessed August 9, 2019.

55. Field A. Discovering Statistics Using IBM SPSS Statistics. 3rd ed. Sage Publications; 2013. 
56. Cohen J. Statistical Power Analysis for the Behavioral Sciences. 2nd ed. Lawrence Erlbaum Associates; 1988. http://www.utstat.toronto.edu/ brunner/oldclass/378f16/readings/CohenPower.pdf.

57. Richardson JTE. Eta squared and partial eta squared as measures of effect size in educational research. Educ Res Rev. 2011;6(2):135-147. doi:10.1016/j.edurev.2010.12.001

58. Walters KL, Horwath PF, Simoni JM. Sexual Orientation Bias Experiences and Service Needs of Gay, Lesbian, Bisexual, Transgendered, and Two-Spirited American Indians. J Gay Lesbian Soc Serv. 2001;13(1-2):133-149. doi:10.1300/J041v13n01_10

59. O'brien K, Tynan A-M, Nixon S, Glazier R. Effectiveness of Progressive Resistive Exercise (PRE) in the context of HIV: systematic review and meta-analysis using the Cochrane Collaboration protocol. BMC Infect Dis. $2017 ; 17$. doi:10.1186/s12879-017- 2342-8

60. O'Brien K, Tynan A-M, Nixon SA, Glazier RH. Effectiveness of aerobic exercise for adults living with HIV: systematic review and meta- analysis using the Cochrane Collaboration protocol. BMC Infect Dis. 2016;16(1):182. doi:10.1186/s12879-016-1478-2

61. Vancampfort D, Mugisha J, Richards J, et al. Dropout from physical activity interventions in people living with HIV: a systematic review and meta-analysis. AIDS Care. 2017;29(5):636-643.

doi:10.1080/09540121.2016.1248347

62. Agarwal RP, Kumar A, Lewis JE. A pilot feasibility and acceptability study of yoga/ meditation on the quality of life and markers of stress in persons living with HIV who also use crack cocaine. J Altern Complement Med. 2015;21(3):152-158 7p. doi:10.1089/acm.2014.0112

63. Mcdermott A, Zaporojan L, Mcnamara P, et al. The effects of a 16-week aerobic exercise programme on cognitive function in people living with HIV. AIDS Care. 2017;29(6):667- 674.

64. Fazeli P, Woods SP, Heaton RK, et al. An active lifestyle is associated with better neurocognitive functioning in adults living with HIV infection. J Neurovirol. 2014;20(3):233-242. doi:10.1007/s13365-014-0240-z

65. Dufour C, Marquine MJ, Fazeli PL, et al. A longitudinal analysis of the impact of physical activity on neurocognitive functioning among hiv-infected adults. AIDS Behav. 2016;22(5):1-11. doi:10.1007/s10461-0161643-z

66. Fazeli P, Marquine M, Dufour C, et al. Physical Activity is Associated with Better Neurocognitive and Everyday Functioning Among Older Adults with HIV Disease. AIDS Behav. 2015;19(8):1470-1477. doi:10.1007/s10461015-1024-z

67. Dufour C, Marquine MJ, Fazeli PL, et al. Physical exercise is associated with less neurocognitive impairment among HIV-infected adults. J Neurovirol. 2013;19(5):410- 417. doi:10.1007/s13365-013-0184-8

68. Mapstone $\mathrm{M}$, Hilton TN, Yang $\mathrm{H}$, et al. Poor aerobic fitness may contribute to cognitive decline in hivinfected older adults. J Am Geriatr Soc. 2013;61(6):S30. doi:10.14336/AD.2013.0400311

69. Monroe A, Zhang L, Jacobson L, et al. The association between physical activity and cognition in men with and without HIV infection. HIV Med. 2017;18(8):555-563. doi:10.1111/hiv.12490

70. Ortega M, Baker LM, Vaida F, et al. Physical activity affects brain integrity in HIV+ individuals. J Int Neuropsychol Soc. 2015;21(10):880-889. doi:10.1017/S1355617715000879

71. Honn VJ, Para MF, Whitacre CC, Bornstein RA. Effect of exercise on neuropsychological performance in asymptomatic HIV infection. AIDS Behav. 1999;3(1):67-74.

72. Northey JM, Cherbuin N, Pumpa KL, Smee DJ, Rattray B. Exercise interventions for cognitive function in adults older than 50: A systematic review with meta-Analysis. Br J Sports Med. 2018;46:71-85. doi:10.1136/bjsports2016-096587 
73. Gomes-Osman J, Cabral DF, Morris TP, et al. Exercise for cognitive brain health in aging: A systematic review for an evaluation of dose. Neurol Clin Pract. 2018;8(3):257-265. doi:10.1212/cpj.0000000000000460

74. Gupta S. Intention-to-treat concept: A review. Perspect Clin Res. 2011;2(3):109-112. doi:10.4103/22293485.83221

75. Brazier A, Mulkins A, Verhoef M. Evaluating a yogic breathing and meditation intervention for individuals living with HIV/AIDS. Am J Health Promot. 2006;20(3):192- 195.

76. Galantino ML, Krafft L, Shepard K, et al. Blending traditional and alternative strategies for rehabilitation: measuring functional outcomes and quality of life issues in an AIDS population. Rehabil Oncol. 1998;16(3):2325

77. Chhatre S, Metzger DS, Frank I, et al. Effects of behavioral stress reduction Transcendental Meditation intervention in persons with HIV. AIDS Care. 2013;25(10):1291-1297. doi:10.1080/09540121.2013.764396

78. Schmid AA, Van Puymbroeck M, Altenburger PA, et al. Poststroke balance improves with yoga: a pilot study. Stroke. 2012;43(9):2402. doi:10.1161/STROKEAHA.112.658211

79. Guner S, Inanici F. Yoga therapy and ambulatory multiple sclerosis Assessment of gait analysis parameters, fatigue and balance. J Bodyw Mov Ther. 2015;19(1):72-81. doi:10.1016/j.jbmt.2014.04.004

80. Schmid A, Van Puymbroeck M, Miller K, Schalk N. P02.166. Group yoga intervention leads to improved balance and balance self- efficacy after stroke. BMC Complement Altern Med. 2012;12:P222. doi:10.1186/1472-6882-12S1-P222

81. Schmid AA, Van Puymbroeck M, Altenburger PA, et al. Balance and Balance Self-Efficacy Are Associated With Activity and Participation After Stroke: A Cross- Sectional Study in People With Chronic Stroke. Arch Phys Med Rehabil. 2012;93(6):1101-1107. doi:10.1016/j.apmr.2012.01.020

82. Ni M, Mooney K, Richards L, et al. Comparative impacts of Tai Chi, balance training, and a specially-designed yoga program on balance in older fallers. Arch Phys Med Rehabil. 2014;95(9):1620-1628.

doi:10.1016/j.apmr.2014.04.022

83. Zbarsky K, Parsley D, Clegg H, et al. Community Balance \& Mobility Scale (CB\&M): Age-related reference values. Physiother Canada. 2010;62 (Suppl).

84. Ramnath U, Rauch L, Lambert E V., Kolbe-Alexander TL. The relationship between functional status, physical fitness and cognitive performance in physically active older adults: A pilot study. PLoS One. 2018;13(4):1-17. doi:10.1371/journal.pone.0194918

85. Won H, Singh DKA, Din NC, et al. Relationship between physical performance and cognitive performance measures among community-dwelling older adults. Clin Epidemiol. 2014;6:343-350.

doi:http://dx.doi.org/10.2147/CLEP.S62392

86. McGough EL, Kelly VE, Logsdon RG, et al. Associations Between Physical Performance and Executive Function in Older Adults With Mild Cognitive Impairment: Gait Speed and the Timed “Up \& Go” Test. Phys Ther. 2011;91(8):1198-1207. doi:10.2522/ptj.20100372

87. Cummings JL, Benson DF. Subcortical Dementia: Review of an Emerging Concept. Arch Neurol. 1984;41(8):874879. doi:10.1001/archneur.1984.04050190080019

88. Elicer I, Byrd D, Clark US, et al. Motor function declines over time in human immunodeficiency virus and is associated with cerebrovascular disease, while HIV- associated neurocognitive disorder remains stable. $J$ Neurovirol. 2018;24(4):514-522. doi:10.1007/s13365-018-0640-6

89. Foley J, Ettenhofer M, Wright MJ, et al. Neurocognitive functioning in HIV-1 infection: effects of cerebrovascular risk factors and age. Clin Neuropsychol. 2010;24(2):265-285. doi:10.1080/13854040903482830 
90. Suresh K. An overview of randomization techniques: An unbiased assessment of outcome in clinical research. $J$ Hum Reprod Sci. 2011;4(1):8-11. doi:10.4103/0974-1208.82352

Figures

\section{O N S O R T}

TRANSPARENT REPORTING of TRIALS

\section{CONSORT 2010 Flow Diagram}

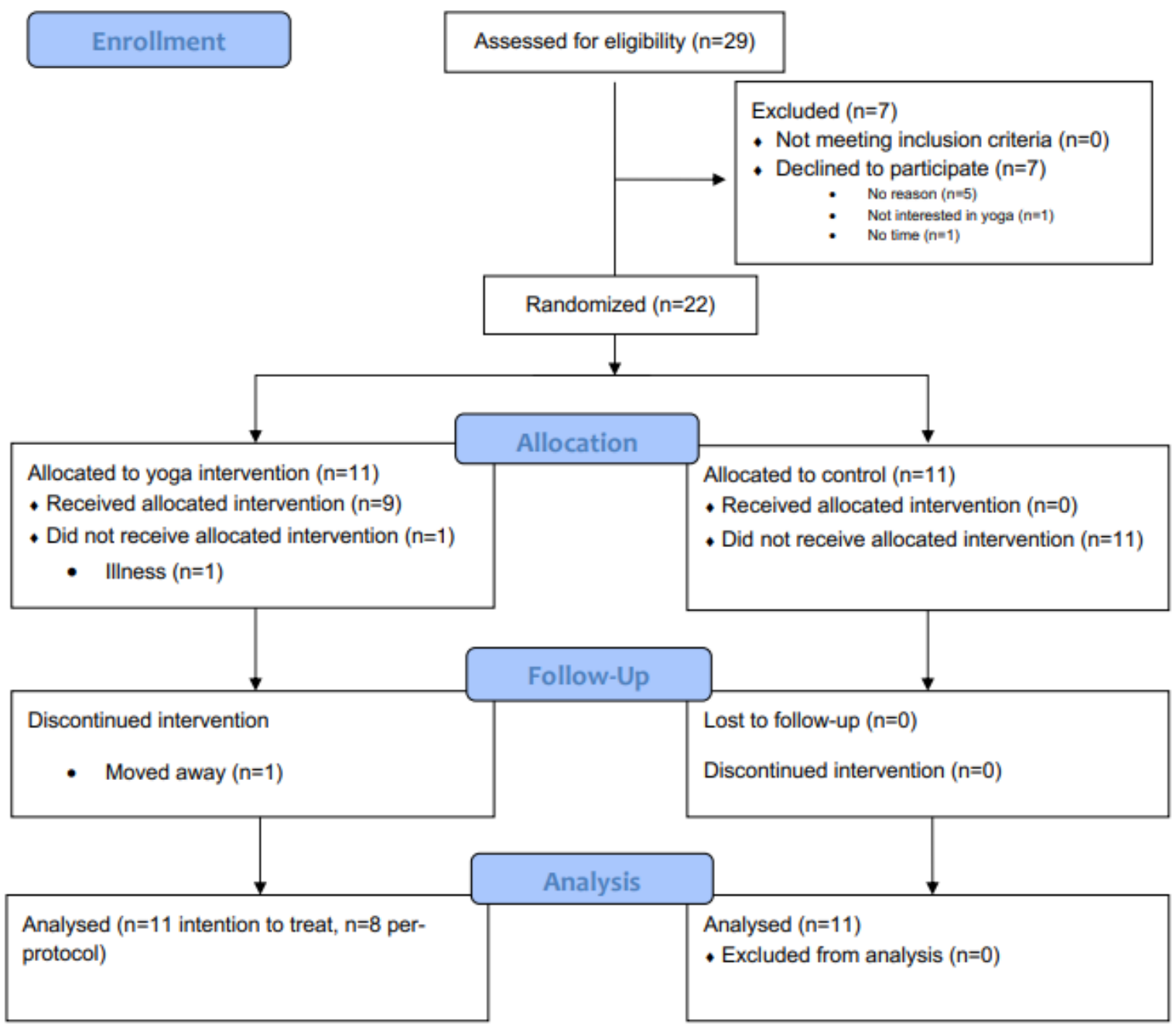

Figure 1

CONSORT Flow Diagram 


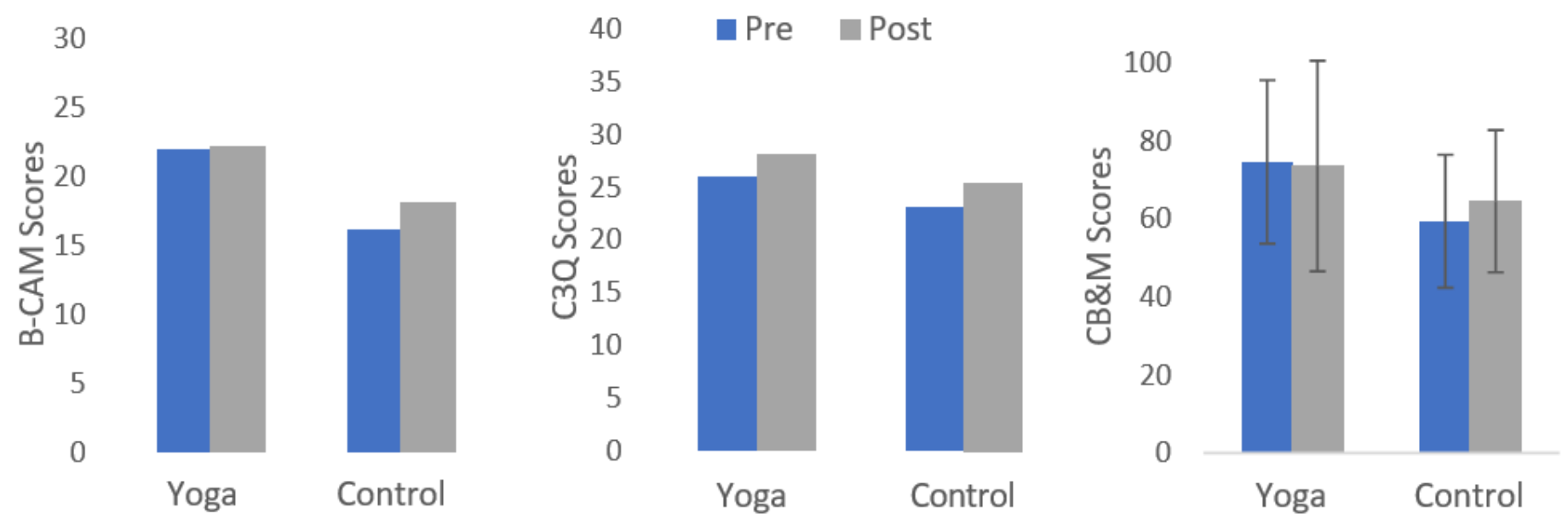

B-CAM, Brief Cognitive Ability Measure; C3Q, Communicating Cognitive Concerns Questionnaire; CB\&M, Community Balance and Mobility Scale

\section{Figure 2}

B-CAM, C3Q, and CB\&M scores pre- and post-intervention.

\section{Supplementary Files}

This is a list of supplementary files associated with this preprint. Click to download.

- CONSORTextensionforPilotandFeasibilityTrialsChecklistmarch212020.pdf 